\title{
UNITARY DISCRETE HILBERT TRANSFORMS
}

\author{
YURII BELOV, TESFA Y. MENGESTIE, AND KRISTIAN SEIP
}

\begin{abstract}
Weighted discrete Hilbert transforms $\left(a_{n}\right)_{n} \mapsto\left(\sum_{n} a_{n} v_{n} /\left(\lambda_{j}-\gamma_{n}\right)\right)_{j}$ from $\ell_{v}^{2}$ to $\ell_{w}^{2}$ are considered, where $\Gamma=\left(\gamma_{n}\right)$ and $\Lambda=\left(\lambda_{j}\right)$ are disjoint sequences of points in the complex plane and $v=\left(v_{n}\right)$ and $w=\left(w_{j}\right)$ are positive weight sequences. It is shown that if such a Hilbert transform is unitary, then $\Gamma \cup \Lambda$ is a subset of a circle or a straight line, and a description of all unitary discrete Hilbert transforms is then given. A characterization of the orthogonal bases of reproducing kernels introduced by L. de Branges and D. Clark is implicit in these results: If a Hilbert space of complex-valued functions defined on a subset of $\mathbb{C}$ satisfies a few basic axioms and has more than one orthogonal basis of reproducing kernels, then these bases are all of Clark's type.
\end{abstract}

\section{INTRODUCTION}

If we are given two finite or infinite sequences of distinct points $\Gamma=\left(\gamma_{n}\right)$ and $\Lambda=\left(\lambda_{j}\right)$ in $\mathbb{C}$ and a sequence of positive numbers $v=\left(v_{n}\right)$, we may define the discrete Hilbert transform by

$$
\left(a_{n}\right)_{n} \mapsto\left(\sum_{n} \frac{a_{n} v_{n}}{\lambda_{j}-\gamma_{n}}\right)_{j}
$$

To make sense of this, we assume that $\Gamma$ and $\Lambda$, viewed as subsets of $\mathbb{C}$, are disjoint. We also assume that $\Lambda$ is a subset of the set

$$
(\Gamma, v)^{*}=\left\{z \in \mathbb{C}: \sum_{n} \frac{v_{n}}{\left|z-\gamma_{n}\right|^{2}}<\infty\right\}
$$

because we wish to define the discrete Hilbert transform for sequences $\left(a_{n}\right)$ in

$$
\ell_{v}^{2}=\left\{\left(a_{n}\right): \sum_{n}\left|a_{n}\right|^{2} v_{n}<\infty\right\} .
$$

If we now associate another weight sequence $w=\left(w_{j}\right)$ with $\Lambda$, we may ask: When is the discrete Hilbert transform $H_{v}(\Gamma, \Lambda)$ given by (1) a unitary transformation from $\ell_{v}^{2}$ to $\ell_{w}^{2}$ ?

The present note answers this question and shows how the solution translates into a general statement about orthogonal bases of reproducing kernels. Making a few minimal assumptions on

2000 Mathematics Subject Classification. 30H10, 46E22.

The authors are supported by the Research Council of Norway grant 185359/V30. 
the underlying Hilbert space, we arrive at the following conclusion: There are no other orthogonal bases of reproducing kernels than those introduced and studied by L. de Branges [4] and D. Clark [3].

\section{Localization OF THE SEQUENCES $\Gamma$ AND $\Lambda$}

Our starting point is the following localization result.

Theorem 1. If the discrete Hilbert transform

$$
H_{v}(\Gamma, \Lambda): \ell_{v}^{2} \rightarrow \ell_{w}^{2}
$$

is unitary, then $\Gamma \cup \Lambda$ is a subset of a circle or a straight line in $\mathbb{C}$.

Proof. In what follows, we let $e^{(n)}$ denote the vectors in the standard orthonormal basis for $\ell_{v}^{2}$. Thus $e^{(n)}$ is the sequence for which the $n$-th entry is $v_{n}^{-1 / 2}$ and all the other entries are 0 .

We fix an index $m$ and observe that since $\Gamma$ is a subset of $(\Lambda, w)^{*}$, the function

$$
G(z)=\left(z-\gamma_{m}\right) \sum_{j} \frac{w_{j}}{\left(\overline{\lambda_{j}}-\overline{\gamma_{m}}\right)\left(\lambda_{j}-z\right)}
$$

is well defined for $z$ in $\Gamma$. In fact, since $H_{v}(\Gamma, \Lambda)$ is assumed to be a unitary transformation, the basis vectors $e^{(n)}$ map into an orthonormal system in $\ell_{w}^{2}$, and therefore $G$ vanishes on $\Gamma$. Thus we may write

$$
G(z)=G(z)-G\left(\gamma_{n}\right)=\left(z-\gamma_{n}\right) \sum_{j} \frac{w_{j}\left(\lambda_{j}-\gamma_{m}\right)}{\left(\overline{\lambda_{j}}-\overline{\gamma_{m}}\right)\left(\lambda_{j}-\gamma_{n}\right)\left(\lambda_{j}-z\right)}
$$

where on the right-hand side we have just subtracted the respective series that define $G(z)$ and $G\left(\gamma_{n}\right)$. It follows that

$$
\frac{G(z)}{z-\gamma_{n}}=\sum_{j} \frac{w_{j}\left(\lambda_{j}-\gamma_{m}\right)}{\left(\overline{\lambda_{j}}-\overline{\gamma_{m}}\right)\left(\lambda_{j}-\gamma_{n}\right)\left(\lambda_{j}-z\right)},
$$

and this function vanishes for $z$ in $\Gamma \backslash\left\{\gamma_{n}\right\}$. Since $H_{v}(\Gamma, \Lambda)$ is assumed to be unitary, the vectors $H_{v}(\Gamma, \Lambda) e^{(n)}$ constitute an orthonormal basis for $\ell_{w}^{2}$, and therefore the sequence

$$
\left(\frac{\lambda_{j}-\gamma_{m}}{\overline{\lambda_{j}}-\overline{\gamma_{m}}} \cdot \frac{1}{\lambda_{j}-\gamma_{n}}\right)_{j}
$$

is a multiple of the sequence $\left(1 /\left(\overline{\lambda_{j}}-\overline{\gamma_{n}}\right)\right)_{j}$. Thus the complex numbers $\left(\lambda_{j}-\gamma_{m}\right)^{2} /\left(\lambda_{j}-\gamma_{n}\right)^{2}$ have the same argument for all $j$, and so

$$
\left(\frac{\left(\lambda_{j}-\gamma_{m}\right)\left(\lambda_{l}-\gamma_{n}\right)}{\left(\lambda_{j}-\gamma_{n}\right)\left(\lambda_{l}-\gamma_{m}\right)}\right)^{2}>0
$$


for $j \neq l$ and $m \neq n$. In other words, the cross ratio of the four complex numbers $\lambda_{j}, \lambda_{l}, \gamma_{n}, \gamma_{m}$ is real. This can only happen if the points lie on the same circle or straight line.

After having applied this argument to four arbitrary points, say $\lambda_{1}, \lambda_{2}, \gamma_{1}, \gamma_{2}$, we see that in fact every point from $\Gamma \cup \Lambda$ lie on the circle or straight line determined by the four initial points, because we may apply the same argument to any given point in $\Gamma \cup \Lambda$ along with three of the points $\lambda_{1}, \lambda_{2}, \gamma_{1}, \gamma_{2}$.

\section{THE UNITARY TRANSFORMATIONS ASSOCIATED WITH $\Gamma$ AND $v$}

For a given sequence $\Gamma$ being a subset of a circle or straight line and an associated weight sequence $v$, we wish to describe those pairs $\Lambda$ and $w$ such that $H_{v}(\Gamma, \Lambda): \ell_{v}^{2} \rightarrow \ell_{w}^{2}$ is a unitary transformation. To begin with, we require the admissibility condition

$$
\sum_{n} \frac{v_{n}}{1+\left|\gamma_{n}\right|^{2}}<\infty
$$

which is now a necessary and sufficient condition for $(\Gamma, v)^{*}$ to be nonempty; we will say that $(\Gamma, v)$ is an admissible pair whenever (2) holds.

We will assume that $\Gamma$ is a subset of the real line. The case when $\Gamma$ is a subset of a circle is completely analogous, as will be briefly commented on at the end of this section. We set

$$
\varphi(z)=\sum_{n} v_{n}\left(\frac{1}{\gamma_{n}-z}-\frac{\gamma_{n}}{1+\gamma_{n}^{2}}\right)
$$

and observe that $\varphi$ is well-defined on $(\Gamma, v)^{*}$ because the series in (3) converges absolutely for $z$ in $(\Gamma, v)^{*}$. We also note that $\varphi$ is a Herglotz function in the upper half-plane ([2], Chapter 9 ). In particular, it is a holomorphic function whose imaginary part is positive. A general Herglotz function $\psi$ in the upper half-plane can be written as

$$
\psi(z)=b+c z+\int_{-\infty}^{\infty}\left(\frac{1}{t-z}-\frac{t}{1+t^{2}}\right) d \mu(t),
$$

where $b$ is a real constant, $c$ a positive constant, and $\mu$ a nonnegative measure on the real line such that

$$
\int_{-\infty}^{\infty} \frac{d \mu(t)}{1+t^{2}}<\infty
$$

We will say that $\psi$ is a purely atomic Herglotz function if $c=0$ and $\mu$ is a purely atomic measure; our function $\varphi$ is thus an example of a purely atomic Herglotz function.

For every real number $\alpha$, we set

$$
\Lambda(\alpha)=\left\{\lambda \in(\Gamma, v)^{*}: \varphi(\lambda)=\alpha\right\} .
$$


We observe that

$$
\sum_{n} \frac{v_{n}(z-w)}{\left(w-\gamma_{n}\right)\left(z-\gamma_{n}\right)}=\varphi(z)-\varphi(w)
$$

which implies that the sequences $\left(1 /\left(\lambda-\gamma_{n}\right)\right)_{n}$ with $\lambda$ in $\Lambda(\alpha)$ constitute an orthogonal set in $\ell_{v}^{2}$. This means that $\Lambda(\alpha)$ is at most a countable set, so that we may associate with $\Lambda(\alpha)$ a weight sequence $w(\alpha)=\left(w_{j}\right)$, where

$$
w_{j}=\left(\sum_{n} \frac{v_{n}}{\left(\lambda_{j}-\gamma_{n}\right)^{2}}\right)^{-1}
$$

for $\lambda_{j}$ in $\Lambda(\alpha)$. It is implicit in our arguments that if $H_{v}(\Gamma, \Lambda): \ell_{v}^{2} \rightarrow \ell_{w}^{2}$ is a unitary transformation, then $\Lambda=\Lambda(\alpha)$ and $w=w(\alpha)$ for some real number $\alpha$.

We will now prove the following theorem.

Theorem 2. Let $(\Gamma, v)$ be an admissible pair with $\Gamma$ a subset of the real line, and let $\alpha$ be a real number. Then the discrete Hilbert transform

$$
H_{v}(\Gamma, \Lambda(\alpha)): \ell_{v}^{2} \rightarrow \ell_{w(\alpha)}^{2}
$$

is unitary if and only if $(\alpha-\varphi(z))^{-1}$ is a purely atomic Herglotz function.

Proof. In this proof, we will again use the standard orthonormal basis vectors $e^{(n)}$ in $\ell_{v}^{2}$; we will denote the corresponding basis vectors in $\ell_{w(\alpha)}^{2}$ by $f^{(j)}$. We will use the notation $\|\cdot\|_{v}$ and $\|\cdot\|_{w}$ for the respective norms in $\ell_{v}^{2}$ and $\ell_{w}^{2}$.

It is clear that the adjoint transformation to $H_{v}(\Gamma, \Lambda(\alpha))$ is again a discrete Hilbert transform. In fact, since $\Gamma$ and $\Lambda(\alpha)$ are sequences of real numbers, we have $H_{v}^{*}(\Gamma, \Lambda(\alpha))=-H_{w(\alpha)}(\Lambda, \Gamma)$, where

$$
H_{w(\alpha)}(\Lambda(\alpha), \Gamma): \ell_{w(\alpha)}^{2} \rightarrow \ell_{v}^{2} .
$$

Therefore, $H_{v}(\Gamma, \Lambda(\alpha))$ is unitary if and only if both $H_{v}(\Gamma, \Lambda(\alpha))$ and $H_{w(\alpha)}(\Lambda(\alpha), \Gamma)$ are isometric. Hence it suffices to check whether $\left(H_{v}(\Gamma, \Lambda(\alpha)) e^{(n)}\right)$ and $\left(H_{w(\alpha)}(\Lambda(\alpha), \Gamma) f^{(j)}\right)$ are orthonormal sequences in respectively $\ell_{w(\alpha)}^{2}$ and $\ell_{v}^{2}$.

The orthogonality of the vectors $H_{w(\alpha)}(\Lambda(\alpha), \Gamma) f^{(j)}$ in $\ell_{v}^{2}$ has already been verified (see (4)); it is just a consequence of the definition of $\Lambda(\alpha)$. Likewise, we have automatically

$$
\left\|H_{w(\alpha)}(\Lambda(\alpha), \Gamma) f^{(j)}\right\|_{v}^{2}=\sum_{n} \frac{w_{j} v_{n}}{\left|\gamma_{n}-\lambda_{j}\right|^{2}}=1 .
$$

So our task is to show that $\left(H_{v}(\Gamma, \Lambda) e^{(n)}\right)$ is an orthonormal sequence in $\ell^{2}(\Lambda(\alpha), w(\alpha))$ if and only if $(\alpha-\varphi(z))^{-1}$ is a purely atomic Herglotz function. 
We first assume that $(\alpha-\varphi(z))^{-1}$ is indeed a purely atomic Herglotz function. It suffices to show that there is a real constant $b$ such that

$$
\frac{1}{\alpha-\varphi(z)}=b+\sum_{j} w_{j}\left(\frac{1}{\lambda_{j}-z}-\frac{\lambda_{j}}{1+\lambda_{j}^{2}}\right)
$$

where $\lambda_{j}$ are the points in $\Lambda(\alpha)$ and $w_{j}$ are as in (5). Indeed, by symmetry, it will then follow that the numbers $\gamma_{n}$ are solutions to the equation

$$
\sum_{j} w_{j}\left(\frac{1}{\lambda_{j}-z}-\frac{\lambda_{j}}{1+\lambda_{j}^{2}}\right)=-b
$$

so that the arguments already employed for the vectors $H_{w(\alpha)}(\Lambda(\alpha), \Gamma) f^{(j)}$ apply similarly to the vectors $H_{v}(\Gamma, \Lambda(\alpha)) e^{(n)}$.

We start from the representation (6), with no a priori assumption on the points $\lambda_{j}$ and the nonnegative numbers $w_{j}$ except the admissibility condition

$$
\sum_{j} \frac{w_{j}}{1+\lambda_{j}^{2}}<\infty
$$

our goal is to prove that the $\lambda_{j}$ are in $\Lambda(\alpha)$ and that the $w_{j}$ are given by (5). We first observe that if we set $z=\lambda_{j}+i y$, then we get, by restricting to imaginary parts,

$$
\frac{w_{j}}{y} \leq\left(\sum_{n} \frac{y v_{n}}{\left(\lambda_{j}-\gamma_{n}\right)^{2}+y^{2}}\right)^{-1}
$$

whence

$$
\sum_{n} \frac{v_{n}}{\left(\lambda_{j}-\gamma_{n}\right)^{2}} \leq w_{j}^{-1}
$$

In other words, the points $\lambda_{j}$ belong to $(\Gamma, v)^{*}$. We now multiply each side of (6) by $z-\lambda_{j}$ and take the limit when $z=\lambda_{j}+i y$ and $y \rightarrow 0^{+}$; since $\lambda_{j}$ is in $(\Gamma, v)^{*}$ and $\varphi\left(\lambda_{j}\right)=\alpha$, this gives (5).

Suppose, on the other hand, that $(\alpha-\varphi(z))^{-1}$ is not a purely atomic Herglotz function and that the vectors $H_{v}(\Gamma, \Lambda(\alpha)) e^{(n)}$ constitute an orthonormal system in $\ell_{w(\alpha)}^{2}$. We will show that this leads to a contradiction. To begin with, our assumption on $(\alpha-\varphi(z))^{-1}$ implies that

$$
\frac{1}{\alpha-\varphi(z)}=b+\sum_{j} w_{j}\left(\frac{1}{\lambda_{j}-z}-\frac{\lambda_{j}}{1+\lambda_{j}^{2}}\right)+c z+\int_{-\infty}^{\infty}\left(\frac{1}{t-z}-\frac{t}{1+t^{2}}\right) d \mu(t)
$$

with $\mu$ a spectral measure such that $\mu\left(\left\{\lambda_{j}\right\}\right)=0$ for every $j$ and not both $c=0$ and $\mu=0$; the fact that the $w_{j}$ are given by (5) can be proved as in the first part of the proof. 
We now argue in the same way as above, reversing the roles of $\Gamma$ and $\Lambda(\alpha)$. This means that we first show, by again restricting to imaginary parts, that

$$
\sum_{j} \frac{w_{j}}{\left(\gamma_{n}-\lambda_{j}\right)^{2}}+\int_{-\infty}^{\infty} \frac{d \mu(t)}{\left(\gamma_{n}-t\right)^{2}} \leq v_{n}^{-1}
$$

for every $n$. We infer from this that both the sum and the integral on the right-hand side of (7) converge absolutely for $z=\gamma_{n}$. Indeed, the right-hand side of (7) vanishes for $z=\gamma_{n}$, and so if we put $z=\gamma_{n}+i \delta$ in (7), divide each side by $i y$, and let $y$ tend to 0 , we get

$$
v_{n}^{-1}=\sum_{j} \frac{w_{j}}{\left(\gamma_{n}-\lambda_{j}\right)^{2}}+\int_{-\infty}^{\infty} \frac{d \mu(t)}{\left(\gamma_{n}-t\right)^{2}} .
$$

Since we should have $\left\|H_{v}(\Gamma, \Lambda(\alpha)) e^{(n)}\right\|_{w(\alpha)}=1$, we have reached a contradiction unless $\mu=0$. On the other hand, if $\mu=0$ and $c>0$, then we also reach a contradiction because the condition for orthogonality of the vectors $H_{v}(\Gamma, \Lambda(\alpha)) e^{(n)}$ becomes

$$
\sum_{j}\left(\frac{w_{j}}{\gamma_{m}-\lambda_{j}}-\frac{w_{j}}{\gamma_{n}-\lambda_{j}}\right)=0
$$

for $m \neq n$, and this is inconsistent with the right-hand side of (7) being 0 whenever $z=\gamma_{n}$.

A few remarks are in order. First, it should be noted that we may have $(\Gamma, v)^{*} \cap \mathbb{R}=\emptyset$ even if $(\Gamma, v)$ is an admissible pair. The following is an example. Pick a sequence of distinct prime numbers $p_{l}$ such that

$$
\sum_{l} p_{l}^{-1 / 2}<\infty
$$

Set $\Gamma=\bigcup_{l} p_{l}^{-1} \mathbb{Z} \backslash \mathbb{Z}$, and equip $\Gamma$ with the weight sequence $v$ obtained by placing a weight of magnitude $p_{l}^{-3 / 2}$ at every point of the sequence $p_{l}^{-1} \mathbb{Z} \backslash \mathbb{Z}$.

On the other hand, if $\Gamma$ is a discrete subset of the real line, then $H_{v}(\Gamma, \Lambda(\alpha)): \ell_{v}^{2} \rightarrow \ell_{w(\alpha)}^{2}$ is unitary for every $\alpha$ with one possible exception: It fails to be unitary when

$$
\sum_{n} v_{n}<\infty \text { and } \alpha=\sum_{n} \frac{v_{n} \gamma_{n}}{1+\gamma_{n}^{2}} .
$$

This statement follows almost immediately from Theorem 2 . We get the exceptional case because the constant $c$ in the representation (7) is obtained as

$$
c=\lim _{y \rightarrow \infty} \frac{1}{i y(\alpha-\varphi(i y))} .
$$

If $\Gamma$ is a subset of the unit circle, then the potential (3) should be replaced by

$$
\varphi(z)=\frac{i}{2} \sum_{n} v_{n} \frac{\gamma_{n}+z}{\gamma_{n}-z}
$$


the analysis goes through in the same way, and we obtain a statement completely analogous to Theorem 2. Note, however, that for discrete sets $\Gamma$ on the unit circle, there will be no exceptional value for $\alpha$ because there is no linear term ' $c z$ ' in the general representation of a Herglotz function. Indeed, a Herglotz function $\psi$ in the unit disk is of the form

$$
\psi(z)=b+\int_{0}^{2 \pi} \frac{e^{i t}+z}{e^{i t}-z} d \mu(t),
$$

where $b$ is a real constant and $\mu$ a nonnegative measure on the circle.

Finally, as will be seen in the last section of this paper, the unitary transformations obtained from Theorem 2 (and its counterpart for the unit circle) correspond precisely to Clark's orthonormal bases [3]. From this point of view, Theorem 2 is essentially a reformulation of Clark's theorem.

\section{ORTHOGONAL BASES OF REPRODUCING KERNELS}

Let $\mathscr{H}$ be a Hilbert space of complex-valued functions defined on some set $\Omega$ in $\mathbb{C}$. To begin with, we assume that $\mathscr{H}$ satisfies the following two axioms:

(A1) If $f$ is in $\mathscr{H}$ and $f(\lambda)=0$ for some point $\lambda$ in $\Omega$, then we may write $f(z)=f_{0}(z)(z-\lambda)$ with $f_{0}$ a function also belonging to $\mathscr{H}$.

(A2) $\mathscr{H}$ has a reproducing kernel $\kappa_{\lambda}$ at every point $\lambda$ in $\Omega$.

We wish to describe those spaces $\mathscr{H}$ which admit orthogonal bases of reproducing kernels. To avoid trivialities, we assume that the dimension of $\mathscr{H}$ is at least 2 . We note that this family of spaces is part of the much larger family of spaces $\mathscr{H}$ that admit Riesz bases of normalized reproducing kernels. Since each space of the latter kind can be equipped with an equivalent norm such that one of the Riesz bases becomes an orthonormal basis, the question of interest is when a space $\mathscr{H}$ has more than one orthogonal basis of reproducing kernels. It is therefore reasonable to introduce a third axiom:

(A3) There exists a sequence of distinct points $\Gamma=\left(\gamma_{n}\right)$ in $\Omega$ such that the sequence of normalized reproducing kernels $\left(\kappa_{\gamma_{n}} /\left\|\kappa_{\gamma_{n}}\right\|_{\mathscr{H}}\right)$ constitutes a Riesz basis for $\mathscr{H}$. In addition, there is at least one point $\lambda$ in $\Omega \backslash \Gamma$ for which $\kappa_{\lambda} \neq 0$.

The Riesz basis $\left(\kappa_{\gamma_{n}} /\left\|\kappa_{\gamma_{n}}\right\|_{\mathscr{H}}\right)$ has a biorthogonal basis, which we will call $\left(g_{n}\right)$. Thus $g_{n}\left(\gamma_{m}\right)=0$ whenever $m \neq n$. We fix an index $n_{0}$ and set $E(z)=g_{n_{0}}(z)\left(z-\gamma_{n_{0}}\right)$. It follows from axiom (A1) that $E(z) /\left(z-\gamma_{n}\right)=g_{n_{0}}(z)+\left(\gamma_{n}-\gamma_{n_{0}}\right) g_{n_{0}}(z) /\left(z-\gamma_{n}\right)$ also belongs to $\mathscr{H}$. We use the suggestive notation $E^{\prime}\left(\gamma_{n}\right)$ for the value of this function at $\gamma_{n}$. We have $E^{\prime}\left(\gamma_{n}\right) \neq 0$ because otherwise $E(z) /\left(z-\gamma_{n}\right)$ would be identically 0 , which can only happen if all functions in $\mathscr{H}$ vanish at every point in $\Omega \backslash \Gamma$; this would contradict the last part of (A3). By 
uniqueness of the biorthogonal sequence $\left(g_{n}\right)$, we now have

$$
g_{n}(z)=\frac{E(z)}{E^{\prime}\left(\gamma_{n}\right)\left(z-\gamma_{n}\right)}
$$

for every $n$. We call $E$, which is unique up to a multiplicative constant, the generating function for $\Gamma=\left(\gamma_{n}\right)$. We may assume that $E$ does not vanish at any point $\lambda$ in $\Omega \backslash \Gamma$, because then $E(z) /(z-\lambda)$ would be a vector in $\mathscr{H}$ vanishing at every point in $\Gamma$. Hence $E(z) /(z-\lambda)$ would be identically 0 , which again would be in contradiction with the second part of (A3).

The sequence $g_{n}$ is also a Riesz basis for $\mathscr{H}$, and therefore every vector $h$ in $\mathscr{H}$ can be written as

$$
h(z)=\sum_{n} h\left(\gamma_{n}\right) \frac{E(z)}{E^{\prime}\left(\gamma_{n}\right)\left(z-\gamma_{n}\right)},
$$

where the sum converges with respect to the norm of $\mathscr{H}$ and

$$
\|h\|_{\mathscr{H}}^{2} \simeq \sum_{n} \frac{\left|h\left(\gamma_{n}\right)\right|^{2}}{\left\|\kappa_{\gamma_{n}}\right\|_{\mathscr{H}}^{2}}<\infty
$$

since point evaluation at every point $z$ is a bounded linear functional, (9) also converges pointwise in $\Omega$. By the assumption that $h \mapsto\left(h\left(\gamma_{n}\right) /\left\|\kappa_{\gamma_{n}}\right\|_{\mathscr{H}}\right)$ is a bijective map from $\mathscr{H}$ to $\ell^{2}$, this means that

$$
\sum_{n} \frac{\left\|\kappa_{\gamma_{n}}\right\|_{\mathscr{H}}^{2}}{\left|E^{\prime}\left(\gamma_{n}\right)\right|^{2}\left|z-\gamma_{n}\right|^{2}}<\infty
$$

whenever $z$ is in $\Omega \backslash \Gamma$. We see that the generating function $E$ appears as a common factor in (9). Since $E(z) \neq 0$ for $z$ in $\Omega \backslash \Gamma$, the function $E$ can be divided out.

We set

$$
v_{n}=\frac{\left\|\kappa_{\gamma_{n}}\right\|_{\mathscr{H}}^{2}}{\left|E^{\prime}\left(\gamma_{n}\right)\right|^{2}}
$$

and observe that since $\Omega \backslash \Gamma$ is assumed to be nonempty, (10) implies that

$$
\sum_{n} \frac{v_{n}}{1+\left|\gamma_{n}\right|^{2}}<\infty
$$

We may now change our viewpoint: Given a sequence of distinct complex numbers $\Gamma=\left(\gamma_{n}\right)$ and a weight sequence $v=\left(v_{n}\right)$ that satisfy the admissibility condition (11), we introduce the space $\mathscr{H}(\Gamma, v)$ consisting of all functions

$$
f(z)=\sum_{n} \frac{a_{n} v_{n}}{z-\gamma_{n}}
$$

for which

$$
\|f\|_{\mathscr{H}(\Gamma, v)}^{2}=\sum_{n}\left|a_{n}\right|^{2} v_{n}<\infty
$$


assuming that the set $(\Gamma, v)^{*}$ is nonempty. The reproducing kernel of $\mathscr{H}(\Gamma, w)$ at a point $z$ in $(\Gamma, v)^{*}$ is

$$
k_{z}(\zeta)=\sum_{n} \frac{v_{n}}{\left(\bar{z}-\overline{\gamma_{n}}\right)\left(\zeta-\gamma_{n}\right)} .
$$

If $\Lambda=\left(\lambda_{j}\right)$ is a sequence in $(\Gamma, v)^{*}$, then we associate with it the weight sequence $w=\left(w_{j}\right)$, where

$$
w_{j}=\left\|k_{\lambda_{j}}\right\|_{\mathscr{H}(\Gamma, v)}^{-2}=\left(\sum_{n} \frac{v_{n}}{\left|\lambda_{j}-\gamma_{n}\right|^{2}}\right)^{-1} .
$$

Consequently, $\left(k_{\lambda_{j}} /\left\|k_{\lambda_{j}}\right\|_{\mathscr{H}(\Gamma, v)}\right)$ is an orthonormal basis for $\mathscr{H}(\Gamma, v)$ if and only if $H_{v}(\Gamma, \Lambda)$ : $\ell_{v}^{2} \rightarrow \ell_{w}^{2}$ is a unitary transformation. Thus from the two previous sections we conclude:

If the space $\mathscr{H}(\Gamma, v)$ has an orthogonal basis of reproducing kernels, then $\Gamma$ is a subset of a straight line or a circle. Moreover, when $\Gamma$ is a subset of the real line, the orthogonal bases of reproducing kernels for $\mathscr{H}(\Gamma, w)$ are obtained from the unitary transformations described by Theorem 2; an analogous result holds when $\Gamma$ is a subset of the unit circle.

\section{Relation to Clark's BASES}

We are now finally prepared to point out the correspondence between our description of unitary discrete Hilbert transforms and the bases studied by de Branges [4] and Clark [3]. We restrict to Clark's bases; the only difference between the two cases is that Clark considered the case of the unit circle while de Branges worked on the real line with, in our terminology, $\left|\gamma_{n}\right| \rightarrow \infty$.

Suppose $\varphi$ is of the form (8) with $\Gamma=\left(\gamma_{n}\right)$ a sequence of distinct points on the unit circle. Then the function

$$
I(z)=\frac{\varphi(z)-i}{\varphi(z)+i}
$$

is an inner function in the open unit disk $\mathbb{D}$. We associate with $I$ the so-called model space $K_{I}^{2}=H^{2} \ominus I H^{2}$, which is the orthogonal complement to the shift-invariant subspace $I H^{2}$ of the Hardy space $H^{2}$ of the unit disk. Since $1 /(1-\bar{\zeta} z)$ is the reproducing kernel for $H^{2}$ at a point $\zeta$ in $\mathbb{D}$, the reproducing kernel for $K_{I}^{2}$ at the same point $\zeta$ is

$$
\kappa_{\zeta}(z)=\frac{1-\overline{I(\zeta)} I(z)}{1-\bar{\zeta} z} .
$$

This formula extends to each point on the unit circle at which every function in $K_{I}^{2}$ has a radial limit whose modulus is bounded by a constant times the $H^{2}$ norm of the function.

A computation shows that

$$
i \frac{1+I(z)}{1-I(z)}=\varphi(z)
$$


which according to Clark's theorem means that the reproducing kernels

$$
\kappa_{\gamma_{n}}(z)=\frac{1-I(z)}{1-\overline{\gamma_{n}} z}
$$

constitute an orthogonal basis for $K_{I}^{2}$. In fact, Clark's theorem says that if $\beta$ is a point on the unit circle and the spectral measure of the Herglotz function

$$
\varphi_{\beta}(z)=i \frac{\beta+I(z)}{\beta-I(z)}
$$

is purely atomic, then the reproducing kernels associated with the spectrum of $\varphi_{\beta}$ also constitute an orthogonal basis for $K_{I}^{2}$. The spectral measures generated in this way correspond precisely to the spectral measures of the functions $1 /(\alpha-\varphi(z))$ with $\alpha$ any real number.

Having observed this correspondence, we conclude that a Hilbert space $\mathscr{H}$ of the type considered in the previous section can have more than one orthogonal basis of reproducing kernels only if $\mathscr{H}$ is, up to trivial modifications, a model space $K_{I}^{2}$ either in the unit disk or in the upper half-plane. ${ }^{1}$

An additional wonder, which can be seen from Clark's theorem or indeed by a straightforward computation, is that the norm in $\mathscr{H}$ can always be computed as an $L^{2}$ integral over a circle or a straight line.

\section{REFERENCES}

[1] Y. Belov, Tesfa Y. Mengestie, and K. Seip, Discrete Hilbert transforms on sparse sequences, arXiv:0912.2899v1, 2009.

[2] J. Cima, A. Matheson, and W. Ross, The Cauchy Transform, Math. Surveys Monogr. 125, Amer. Math. Soc., Providence, RI, 2006.

[3] D. N. Clark, One dimensional perturbations of restricted shifts, J. Analyse Math. 25 (1972), 169-191.

[4] L. de Branges, Hilbert Spaces of Entire Functions, Prentice-Hall, Englewood Cliffs, 1968.

\footnotetext{
${ }^{1}$ It may be objected that we fall short of characterizing all Clark bases because there exist model subspaces that do not satisfy axiom (A1) above. However, axiom (A1) can be relaxed in an appropriate manner so that this shortcoming is removed; we refer to our recent paper [1] for details.
} 
Department of Mathematical Sciences, Norwegian University of Science and Technology (NTNU), NO- 7491 TRONDHEIM, NORWAY

E-mail address: j_b_juri_belov@mail.ru

Department of Mathematical Sciences, Norwegian University of Science and Technology (NTNU), NO- 7491 TRONDHEIM, NORWAY

E-mail address: mengesti@math.ntnu.no

Department of Mathematical Sciences, Norwegian University of Science and Technology (NTNU), NO- 7491 TRONDHEIM, NORWAY

E-mail address: seip@math.ntnu.no 